\title{
Fingerprint Scan of The Dead: Real-Time Identification During Search and Recovery Phase in Large Scale Disaster
}

Research Article

Lay See Khoo ${ }^{1 *}$, Poh Soon Lai ${ }^{1}$, Muhammad Syafiq Zamasry ${ }^{1}$, Ahmad Hafizam Hasmi ${ }^{1}$, Mansharan Kaur ${ }^{2}$, Hapizah Md Nawawi², Mohamad Azaini Ibrahim $^{1}$, Mohd Shah Mahmood ${ }^{1}$

${ }^{1}$ National Institute of Forensic Medicine (NIFM), Hospital Kuala Lumpur, Ministry of Health Malaysia, Malaysia.

${ }^{2}$ Institute of Pathology, Medical \& Forensic Laboratory (iPERFORM),Universiti Teknologi MARA (UiTM), Malaysia.

\section{Abstract}

Fingerprint analysis have been the gold standard for personal identification and a most reliable means of primary identification in Disaster Victim Identification (DVI) work. However, finger printing of the dead has always been primarily performed in the mortuary during postmortem (PM) examination. This identifier maybe lost due to the transportation and body handling where the duration of the recovery of dead bodies could exceed 48 hours and the bodies have already started to decompose. The objective of the study is to demonstrate the possibility of using a portable biometric reader to capture and to match with a cadaveric fingerprint scanin order to translate into real-time identification in a large scale disaster. The authors used an optical fingerprint biometric reader MSO 1350 and a smartphone to conduct this study. An initial pilot study of 18 subjects and a total of 100 known deceased were used to match ownthumbprintswith theirMalaysian Identity Card (MyKad).Descriptively, 86.9\% of the cadaveric finger prints were matched. Inferential statistical analysis was performed using IBM SPSS Version 26.0. The analysis of the data proved that the fingerprint biometric reader device and its application which has been used for the living can also be used for the dead. Limitation of the study was also discussed. This study highlights that rapid victim identification through real-time fingerprint comparison is possible using a portable fingerprint biometric reader.Management of the dead is part of the DVI process whereby first responders are the main component in the Scene phase. Ideally, first responders are the best resources to be effectively maximised and trained to retrieve fingerprint informationin order to preserve identity during the search and recovery for the dead in a large scale disaster. The conventional fingerprinting of the dead in the PM phase can now be brought forward to the Scene phase by advancing digital technology as a paradigm shift in the DVI process. Immediate capture of the biometrics of dead victims have ushered in a new dimension in humanitarian forensic action to access means of protecting the identity and dignity of the dead.

Keywords: Real-TimeIdentification; Cadaveric Fingerprint Scan; Portable Biometric Reader; First Responders; Large Scale Disaster; DVI; Humanitarian Forensic Action.

List of Abbreviations: AFIS: Automated Fingerprint Identification System; AM: Antemortem; DVI: Disaster Victim Identification; FMIS: Forensic Medicine Information System; HKL: Hospital Kuala Lumpur; ICRC: International Committee of the Red Cross; MREC: Medical Research Ethical Committee; MyKad: Malaysian Identity Card; NMRR: National Medical Research Register; PM: Postmortem; PPE: Personal Protective Equipment; RMP: Royal Malaysia Police; SOP: Standard Operating Procedure; USB: Universal Serial Bus.

\section{Introduction}

According to Khoo et al. (2018), the 'Golden 48 hours' rule, which is the first 48 hours' post-disaster before the decomposition process commences is of ultimate value to the forensic pathologist where potential identifiable characteristics from the dead can be documented and prevented from loss, right before the decomposition process begins [1]. Thus, the immediate capture of the biometrics of the dead victims in-situ is necessary to be performed during the post-disaster search and recovery phase. The biometric system is an automatic identification and authentication system that uses unique biological traits such asthe fingerprint, face, iris,

*Corresponding Author:

Khoo Lay See,

National Institute of Forensic Medicine, Hospital Kuala Lumpur, Jalan Pahang, 50586 Kuala Lumpur, Malaysia.

E-mail: khoolaysee@yahoo.com

Received: January 24, 2022

Accepted: February 09, 2022

Published: February 11, 2022

Citation: Lay See Khoo, Poh Soon Lai, Muhammad Syafiq Zamasry, Ahmad Hafizam Hasmi, Mansharan Kaur, Hapizah Md Nawawi, et al.,. Fingerprint Scan of The Dead: RealTime Identification During Search and Recovery Phase in Large Scale Disaster. Int J Forensic Sci Pathol. 2021;9(2):475-482. doi: http://dx.doi.org/10.19070/2332-287X-2200099

Copyright: Khoo Lay See ${ }^{\circ}$ 2022. This is an open-access article distributed under the terms of the Creative Commons Attribution License, which permits unrestricted use, distribution and reproduction in any medium, provided the original author and source are credited. 
voice, retina etc. of an individual. Of all these systems, the fingerprint biometric system is the most widely used because of its low cost, high matching speed, and relatively high matching accuracy [2] whereby it has become a trusted authentication mechanism in the health-care, financial, retail, education, manufacturing, military, and law enforcement sectors used in applications for the benefit of society such as tracking child vaccination schedules, identifying missing children, and preventing fraud in food subsidies $[2,3]$. The fingerprint biometric system is a touch-based recognition system, with comparatively small finger surface exposed to the sensor for feature extraction and comparison, thus leading to relatively high matching speed and accuracy with moderate cost. It is a pattern recognition technique that recognizes a person based on a feature vector extracted from the person's fingerprint pattern [4]. This paper demonstrates the possibility to perform Disaster Victim Identification (DVI) on-site with the concept of real-time fingerprint matching of the dead using a mobile and portable fingerprint biometric device where the thumbprint of the dead is readable with in a given timeframe. Immediate identification enhances the DVI process and allows a human itarian approach to the handling of the dead and their remains, bringing much needed closure to families and their loved ones.

\section{Methodology}

\section{Material}

For thisstudy, the authors have used an optical fingerprint biometric reader, called the MorphoSmartmode IMSO 1350 (Figure 1). Optical technology offers superior image quality and this model is light and portable, and at the same time, it can be connected to an android-based smartphone device via a Universal Serial Bus (USB) cable, which simulates the mobile environment the authors are looking for. Some biometric readers available in the market can only be connected to a laptop or computer desktop, which is not suitable for this study. The MSO 1350 will be paired with the fingerprint application software version no. 2.0.7 developed by Kridentia Tech SdnBhd which is installed in an android Huawei
P9 smartphone model EVA L19 used to display the information (Figure 2).

\section{Field site}

The data were collected in the Department of Forensic Medicine, Hospital Kuala Lumpur (HKL)as perthe department's Standard Operating Procedure (SOP) [5]. It is standard practice to wear basic personal protective equipment (PPE) such as gloves, disposable apron and face mask during the fingerprint matching processof the deceased.

\section{Subjects}

A simple experimental study to engage 100 known deceased with available Malaysian Identity Card (MyKad)to match with ownthumbprints. This is to say all non-medicolegal or non-police caseswhere the deceasedare notsubjectedto a postmortem examination, are included in thestudy [5]. Non-Malaysians and cases of unknown identity as well as decomposed bodies are excluded. MyKad is an identification card for Malaysian citizen aged 12 and above that incorporates both photo identification and fingerprint biometric data on an in-built computer chip embedded in a piece of plastic issued by the National Registration Department of Malaysia. MyKad stores the following information, i.e. name, address, race,citizenship status and fingerprint minutiae [6]. By using a $\mathrm{MyKad}$ reader, fingerprint verification can be done accurately [6].

\section{Compliance with Ethical Standards}

This study is registered under the National Medical Research Register (NMRR) with registration number NMRR-19-114048272(IIR). Ethical approval must then be obtained from the Director of Hospital Kuala Lumpur and the Medical Research Ethical Committee (MREC), Ministry of Health Malaysia for any study involving human subjects. The approval letter was obtained dated 2 July 2019 with MREC number KKM/NIHSEC/P19-

Figure 1. MSO 1350.

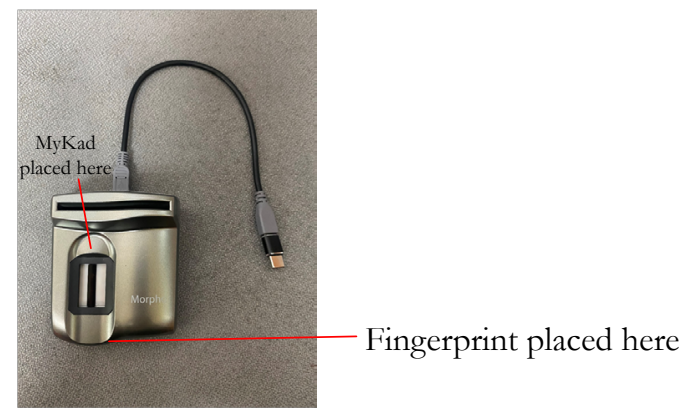

Figure 2. Huawei P9 smartphone model EVA L19.

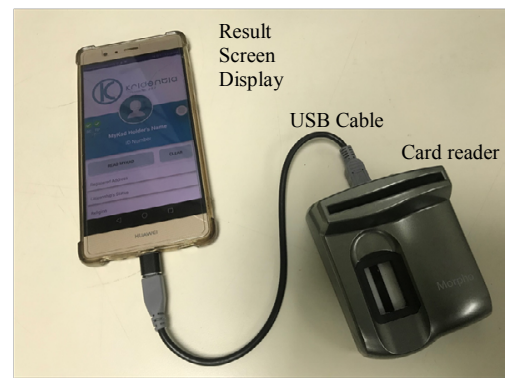


1166(12). This research has only required to use the thumbprint of the known dead body to match it with its own Malaysian Identity Card (MyKad). There was thusno harm inflicted on the dead body whereby no dissection was performed on the subject and neither tissues nor organs were removed. Conflict of Interest: The authors have declared no conflict of interest.Informed consent: Consent from the next-of-kin will be obtained by the research team using a consent form upon releasing of the body at the registration counter. Next-of-kin will then provide the MyKad of the deceased to match with the thumbprint of the deceased. The simple protocol of thumbprint matching is explained to the nextof-kin or relatives. Privacy \& Confidentiality: All data obtained are strictly for the use of the research development. Information on MyKad will not be stored in the device. The MorphoSmartmodelMSO 1350 fingerprint biometric reader and application software are only for matching and display of information. The application software does not have any storage capacity.

\section{Study design and duration}

A cross sectional study performed in the Department of Forensic Medicine, Hospital Kuala Lumpur for a period of 12 months from 1 January 2020 to 31 Dec 2020. However, a pilot study was done from 1 August to 31 December 2019 after ethical approval was granted in July 2019 in order to get a correct sample size for this study.

Pilot study: There are a number of settings when undertaking a pilot trial when there is no prior information to base a sample size on. For such pilot studies, the recommendation is a sample size of 12 to 35 per group $[7,8]$. Hence, in thisstudy a total of 18 subjects were chosen to determine the unknown population proportion, p.As per Table 1 , there were 14 matched fingerprint scans from 8 males of an average age of 59 years old and 6 females of an average age of 50 years old. There were 4 unmatched fingerprint scans from 4 females of an average age of 79 years old. From here, the unknown population proportion, pis $14 / 18=0.78$.

Sample size: A sample is any part of the population, usually a subset of a population, whose properties have been, or are to be generalized to the population. The correct sample size to use is important in research to test the hypothesis about the population from which the samples are drawn, i.e. the subjects from the death in ward cases [9]. Sample size is arrived at through statistical calculations.In this study where the plan is to estimate the proportion of successes in a dichotomous outcome variable which is either Matchedor Not matched fingerprint scan in a single population, the formula for determining sample size is as below; where $\mathrm{Z}$ is the value from the standard normal distribution reflecting the confidence level that will be used ( $\mathrm{Z}=1.96$ for $95 \%)$ and $\mathrm{E}$ is the desired margin of error of 0.1 . The authors are planning a study to generate a $95 \%$ confidence interval for the unknown popula- tion proportion, $\mathrm{p}$.

$$
\begin{aligned}
& n=p(1-p)\left(\frac{Z}{E}\right)^{2} \\
& \mathrm{n}=0.78(0.22)(1.96 / 0.1)^{2} \\
& \mathrm{n}=66
\end{aligned}
$$

The minimum sample requirement are 66 subjects from the death in ward cases. For this study however, the authors collected a total of 100 fingerprint scans for this study.

Sampling method: A simple random sampling is used in thisstudy. This is abasic sampling technique where a group of subjects (a sample) isselected from a larger group (a population). Each individual is chosen entirely by chance and each member of the population has an equal chance of being included in the sample [9]. In this case, 100 subjects from thedeath in ward cases were sampled to match their fingerprints using the fingerprint biometric reader MSO 1350.

\section{Data Collection Flow}

Death-in-ward cases are deaths which occur in the ward and where causes of death are known and given by the attending ward doctor. The time of death is available and recorded by the attending doctor. Upon arrival at the Department of Forensic Medicine, HKL the case is registered into the Forensic Medicine Information System (FMIS) and the authors are then informed by the staff on duty. A running case number is given to the subject and consent is obtained from the next-of-kin upon claiming of the body at the registration counter. The MyKad of the subject is matched with the subject's own thumbprint to verify the identity. The time of death, the time the body arrived at the department and the time the thumbprint was taken will be noted in order to see the time interval involved. The whole matching process took approximately 1 to 2 minutes. The matched result of the thumbprint scan image with the subject's MyKadis noted. The body is then released to the next-of-kin (Figure 3 and 4).

\section{Statistical Analysis}

The analysis is performed using the IBM SPSS (Statistical Package for Social Sciences) Version 26.0. The data are manually entered into the software, followed by a cleaning process to prevent errors that may skew the results prior to analysis. Descriptive analysis is used for selected variables based on distribution. Numerical data and continuous variables are presented as mean and standard deviation if normally distributed or medians and interquartile ranges if not normally distributed.

Null Hypothesis: Male and female are equally likely to get a

Table 1. Pilot study result.

\begin{tabular}{|c|c|c|c|c|}
\hline NO. & Mean age (years) & Gender & Output scan & Total \\
\hline 1 & 59 & Male & Matched & 8 \\
\hline 2 & 50 & Female & Matched & 6 \\
\hline 3 & 79 & Female & Not matched & 4 \\
\hline & & & & 18 \\
\hline
\end{tabular}


fingerprint match on the result output of the biometric scan

The relationship between the two categorical variables were evaluated by Chi Square test with Spearman Correlation which is also a non-parametric test due to the fact that the population data does not have a normal distribution curve.

Null Hypothesis: There is no relationship among age groups and the result output of the biometric scan

The relationship between the two categorical variables were evaluated by Chi Square test with Spearman Correlation which is also a non-parametric test due to the fact that the population data does not have a normal distribution curve. The subjects were divided into four age groups for this study; i.e. 21-40, 41-60, 61-80 and 81-100 years old.

Null Hypothesis: There is no relationship between the duration from time of death to the time of scan performed with the result output of the biometric scan

A non-parametric test of Mann-Whitney U test and Spearman Correlation were used to evaluate two independent variables, i.e. the duration from time of death to the time of scan performed.
Logistic Regression: All the three parameters i.e. gender, age group and duration from time of death to the time of scan performed with the binary result output of the biometric scan

To evaluate relationship among all the variables that may be contributing to affect the binary result output of the biometric scan.

\section{Findings}

There were a total of 100 randomized selected subjects i.e. 56 males and 44 females for the fingerprint scanning by using their respective Malaysian Identity Cards (MyKad); however, one female subject was excluded from this study due to a faulty chip in her MyKad, whereby there was no output of scan result available. Descriptively, there were a total of 52 males and 34 females which matched (86.9\%) and 4 males and 9 females that did not match (13.1\%). As shown in Table 2, males achieved a higher percentage of fingerprint matching at $92.9 \%$ compared to females at only $79.1 \%$ with a difference of $13.8 \%$. The Pearson Chi square test showed $\mathrm{X}^{2}(1, \mathrm{~N}=99)=4.053, \mathrm{p}=0.044$ and the expected cell frequency was more than 5 which has not violated the main assumption of chi square. Nevertheless, the Spearman correlation was used and it proved that there was a very weak relationship between gender and result output of biometric scan at $\mathrm{r}(99)=$

Figure 3. Flow chart of the data collection.

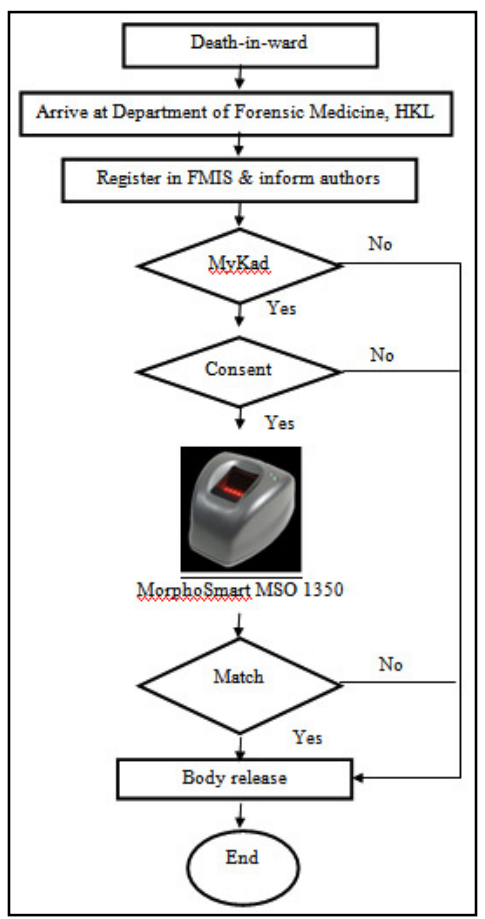

Figure 4. Fingerprint matching process.

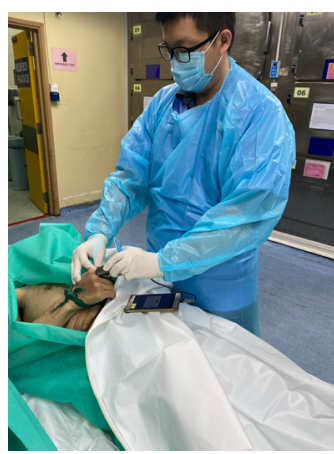


- .202, $\mathrm{p}=0.045$. Furthermore, logistics regression showed that gender was not a significant variable $(p>0.05)$ that really affected the binary result output of the biometric scan.

The median age of the subjects included in this study was 67 \pm 21 years old. The subjects were further divided into four age groups for this study as displayed in Table 3. The age group of 4160 years recorded the highest percentage at $96.7 \%$ of fingerprint matching as compared to the other age groups whereby the lowest was within the age group of $81-100$ years at only $64.3 \%$. Most of the mismatched biometric scans of subjects were more than 60 years old at $76.9 \%$ and only 3 subjects were less than 60 years old. The Pearson Chi square test showed $X^{2}(3, N=99)=9.001$, $\mathrm{p}<0.05$, the expected cell frequency however was only $1.58(<5)$ and has violated the main assumption of chi square. Hence, the Spearman correlation was used and demonstrated that there was no relationship between age group and result output of biometric scan at $\mathrm{r}(99)=-.192, \mathrm{p}>0.05$. Furthermore, logistics regression was performed and showed that age group was also not a significant variable $(p>0.05)$ that could affect the binary result output of the biometric scan.

The median post-mortem interval of the subjects included in this study was $158 \pm 129$ minutes as shown in Table 4. The MannWhitney U Test showed no significant differences in the postmortem interval based on the fingerprint matching output, $\mathrm{z}=$ - 1.321, p > 0.05. Based on the Spearman correlation, there was also no relationship between post-mortem interval and result out- put of biometric scan at $\mathrm{r}(99)=-.302, \mathrm{p}>0.05$. Furthermore, logistics regression showed that post-mortem interval was definitely not a significant variable $(\mathrm{p}>0.05)$ that might affect the binary result output of the biometric scan.

Based on the logistic regression analysis, the relationship among all the three variables including gender, age group and post-mortem interval that may be contributing to affect the binary result output of the biometric scan were proven to be not significant at $\mathrm{p}>0.05$ whereby their respective $\mathrm{p}$ values were $\mathrm{p}=0.080, \mathrm{p}$ $=0.052$ and $\mathrm{p}=0.954$. The analysis of the data has proven that fingerprint biometric reader devices and its application which has been used for the living can also be used for the dead. Hence it supports the assumption that real-time fingerprints can scan assist inidentification for disaster related casualties. The use of fingerprint scan in post-disaster events or during recovery of remains will improve on the cadaveric fingerprint collection for rapid victim identification.

\section{Discussion}

The use of friction ridge prints (fingerprints, palm prints or footprints) analysis or fingerprint comparisonis often the fastest method and most reliable means of primary identification indisaster victim identification (DVI) work [10,11]. The DVI process comprising the Scene, the Postmortem (PM), the Antemortem (AM) and the Reconciliation phase, is an internationally recognized sequence of activities that is used to collectively describe the pro-

Table 2. Number of subjects based on gender according to the result output of the biometric scan.

\begin{tabular}{|c|c|c|c|c|}
\hline \multirow{2}{*}{ Variables } & \multicolumn{2}{|c|}{ Fingerprint Matching } & \multirow{2}{*}{ Total } \\
\cline { 3 - 4 } \multicolumn{2}{|c|}{} & \multicolumn{2}{|c|}{ Number (\%) } & \\
\cline { 2 - 4 } & Match & Not Match & \\
\hline \multirow{2}{*}{ Gender } & Male & $52(92.9)$ & $4(7.1)$ & 56 \\
\cline { 2 - 4 } & Female & $34(79.1)$ & $9(20.9)$ & 43 \\
\hline \multicolumn{2}{|c|}{ Total } & $86(86.9)$ & $13(13.1)$ & 99 \\
\hline
\end{tabular}

Table 3. Number of subjects based on age groups according to the result output of the biometric scan.

\begin{tabular}{|c|c|c|c|c|}
\hline \multicolumn{2}{|c|}{ Variables } & \multicolumn{2}{c|}{ Fingerprint Matching } & \multirow{2}{*}{ Total } \\
\cline { 3 - 4 } \multicolumn{2}{|c|}{} & \multicolumn{2}{c|}{ Number (\%) } & \\
\cline { 2 - 4 } & Match & Not Match & \\
\hline Age Groups & $21-40$ & $10(83.3)$ & $2(16.7)$ & 12 \\
\hline (Years) & $41-60$ & $29(96.7)$ & $1(3.3)$ & 30 \\
\hline & $61-80$ & $38(88.4)$ & $5(11.6)$ & 43 \\
\hline & $81-100$ & $9(64.3)$ & $5(35.7)$ & 14 \\
\hline \multicolumn{2}{|c|}{ Total } & $86(86.9)$ & $13(13.1)$ & 99 \\
\hline
\end{tabular}

Table 4. Descriptive statistic of age and post-mortem interval of the subjects.

\begin{tabular}{|c|c|c|}
\hline & $\begin{array}{c}\text { Age } \\
\text { (Years) }\end{array}$ & $\begin{array}{c}\text { Post-mortem In- } \\
\text { terval (Minutes) }\end{array}$ \\
\hline Minimum - Maximum & $23-91$ & $64-10590$ \\
\hline Mean \pm SD & $62.49 \pm 16.27$ & $325.88 \pm 1055.68$ \\
\hline Median \pm Interquartile Range & $67.00 \pm 21.00$ & $158.00 \pm 129.00$ \\
\hline Normality Test & $\mathrm{p}<0.05$ & $\mathrm{p}<0.05$ \\
\hline
\end{tabular}


cess in which victims of a mass fatality are identified [10]. Kaushal $\&$ Kaushal [12] have mentioned that the fingerprint identification is the oldest forensic discipline known to man and it has proved over time to be the most rapid, reliable and cost-effective means by which to identify unknown deceased individuals especially in mass disasters. Each individual possesses a unique set of minute raisedridges on volar pads called 'friction ridge skin'. These clear and apparent unique outlines of the ridges are called fingerprints. Fingerprints are unique and highly individualistic across individuals by virtue of discriminate features forming the basis for personal identification in forensic examinations [13]. A fingerprint can be described as a combination of ridges and valleys on the skin. Ridges and valleys form patterns known as arches, loops and whirls as described by Kawagoe and Tojo [14] and by Moayer and $\mathrm{Fu}$ [15] which were both extended on Henry's system of fingerprint classification. Fingerprints, along with forensic dental comparison andDNA analysis, are paramount primary identifiersin the identification of unknown deceased individuals and human remains [16].

Optical scanners are the most commonly used law enforcement capture platforms. They have the most robust development since they have been used the longest and are the basis behind most traditional "livescan" systems [11]. Optical scanners work by reflecting light off of the finger that has been placed on an optical plate and use a computer sensor to read the differences in contrast among the ridges and furrows to create a two-dimensional representation of the fingerprint [17]. In thisstudy, the authors usingan optical fingerprint biometric reader, called the MorphoSmart model MSO 1350 have managed to prove that fingerprint identification for the dead yield the same results as it does for the living. Thisis supported by a study by Johnson \& Riemen [11], whereby fingerprint use in the identification of a decedent is no different than identifying a live person. In other words, the opportunity to explore real-time identification using fingerprint scan for the dead during the search and recovery phase in a large scale disaster can be realized into best operational practices for first responders. First responders are trained and skilled personnel, working with disaster response agencies and directly involved in on-site disaster scene. They work within the disaster zone and are tasked with both search and rescue of the injured and survivors as well as making up the search and recovery team for dead bodies' retrieval. A robust fingerprint device and its application can be made suitable to be used by first responders in disaster related casualties. The use of fingerprint scanning within 48 hours of a disaster event or recovery of remains will improve the relevant information collected for rapid identification.

In Malaysia, inquiries into deaths fall within the jurisdiction of the Royal Malaysia Police (RMP) and the mandate to conduct postmortem examinations on dead bodies is provided for in the Criminal Procedure Code, i.e. to a Government Medical Officer particularly a forensic pathologist [18]. It is not the norm for forensic pathologist to be called to a disaster site or scene of incident and forensic pathologists usually conduct forensic examinations at the mortuary unless the RMP requires them to be on site. As a standard practice, dead victims will be retrieved by the first responders and they will be transported by the police to the mortuary for examination. By the time the bodies arrive at the mortuary, evidence that could be used to facilitate identification might be lost due to the transportation and body handling. Similarly, the duration of the recovery of the bodies could exceed 48 hours and the remains may have already started to decompose. Capturing PM cadaveric fingerprints in the mortuary to match the AM data can be very challenging to the ridgeologist, a practitioner who collects and compares known and questioned friction ridge prints to effect an identification or individualization.

Mass disasters with a large number of unknown victims are among the biggest challenges for the forensic disciplines and identification has always been one of the important roles of the forensic pathologist. Fingerprint impression is usually taken in the mortuary during PM examination in all disasters. For instance, a total of 37 Danish victims $(29.7 \%$ of the cases)were matched by using fingerprints following the December 2004 tsunami in Thailand [19]. Overall, out of 2,679 identified victims, fingerprints were used to establish identity in 670 cases $(25.0 \%$ ) [20] whereas there was a $60 \%$ hit rate on fingerprints for Thai victims only asall Thais over the age of 15 possess identification cards bearing their fingerprints [21]. The rate of successful identification could have been much improved with the use of a fingerprint reader device during the recovery of the human remains at the DVI Scene phase.A study by Gahr et al. [22] has supported the concept of the 'Golden 48 hours' rule, whereby the potential identifiable characteristics, in this casethe fingerprint,can be recorded immediately, right before the decomposition process begins within the first 48 hours' postdisaster. The German authors conducted a study on 400 fingerprints retrieved from bodies in various states of decay which have undergone than atopractical processing that allows morphological reconstruction of very advanced decomposed bodies. Despite the complicated fingerprint treatment called than atoprint of the hand region in than atopractical processing, the chances of matching the fingerprints for positive identification from AFIS (Automated Fingerprint Identification System) showeda downward curve and this finding strengthened the concept of the Golden 48 hoursto record the identifiers before they are lost [23].

Other than the digital fingerprint scanner, there are many other techniques available for fingerprint impressionsretrieval from the dead bodies including powder and tape, ink pad techniques, melted paraffin wax and oils, hot water as well as heating procedure [22, 23]. Simple techniques such as ink pad techniques could also be easily performed by the first responders at the Scene phase whereby fingerprints retrieved can be fixed on the dedicated fingerprint index cards and photographed immediately by using a digital camera or smartphone and subsequently transferred to the data centre for immediate fingerprint comparison through a phone application [23]. In fact, latest drone technologies can be adopted to assist DVI work to link fingerprint impressions taken on-site to data centre for fingerprint comparison purpose. In this manner, weight-carrying drone with payload capacity could be used to transport print impression index cards to the DVI command centreas an alternative to consider. Nevertheless, more data and research are needed for the head start.

To recapitulate the findings from the current study, the fingerprint biometric reader has shown to have a significant outcomein its application in the management of the dead in large scale disasters. Given that first responders are easily accessible and are a readily available task force in the field of managing disasters, particularly in the Scene phase of the search and recovery of human remains, they can be trained and their effectiveness maximised to retrieve fingerprint information before these are permanently lost. It is hoped the findings from this study can be shared with all first re- 
sponders' agencies in whichthe use of a portable fingerprint biometric reader can be incorporated in their SOP during the search and recovery of dead victims. This can be taken as initial phase of first responders task in managing the dead and subsequently protecting the dignity of the dead. In ensuring effective and efficient traceability and identification of the dead, capturing real-time cadaveric fingerprints could bean addition to the field manual for first responders in management of dead bodies [24].

\section{Limitation of the Study}

In this study, the subjects were all placed under a controlled environment and kept inside body freezers at temperature of $2-4^{\circ} \mathrm{C}$ prior to release to their respective next-of-kin. Hence, there is a slightshortcoming in representing a real disaster situation. In addition, the MorphoSmart model MSO 1350 is only applicable to read the Malaysian Identity Card (MyKad) upon matching the fingerprint of a Malaysian citizen. To elaborate further, foreigners were also excluded from this study whereby in an actual mass fatality incidentsuch as a bus accident or aircraft crash, victims usually comprise of a mixture of nationalities. That is to say, identification willbe an arduous task for the DVI team and thus a more sophisticated biometric reader device needs to be developed to capture facial images for recognition [25] as well as to record and store cadaveric fingerprints for subsequent matching.

\section{Conclusion}

Conventionally, fingerprinting of the dead is usually undertaken by the scene of crime or fingerprint officers in the mortuary during the postmortem (PM) examination. From this study, the authors have proved that the recovery of fingerprints from the dead is possible within 48 hours using an optical fingerprint biometric readerused for the living. Hence a handheld portable fingerprint biometric reader can be applicable at the scene of death orin large scale disasters to enable rapid real-time fingerprint comparison. Fundamentally, managing human remains which include preserving their dignity as well as helping to identify them, is one of the three pillars of humanitarian response following conflicts or disasters [26]. Under this umbrella of humanitarian forensic action, retrieval of victim identity can now be enhanced through the in-situfingerprint matchingat the disaster site which can be definitely performed within the Golden 48 hours. Potential identifiable characteristics from the dead particularly the fingerprints can be documented in-situ and prevented from loss by advancing the PM phase to the Scene phase in DVI.

As there is no current method available to identify dead bodies using a biometric reader at a disaster site, this study will allow an almost immediate identification for all dead victims before the remains are transported to the mortuary. Immediate capture of the biometrics of the dead victims as means of protecting the identity and dignity of the dead person is the new normal in humanitarian forensic action. First responders are the main component during search and recovery in the Scene phase of DVI. With a proper mobile fingerprint biometric reader device given to first responders, the conventional fingerprinting process in the mortuary can be brought forward to the Scene phase to allow for preservation of identity. This improvised DVI process andthe new operational SOP for first responders to capture fingerprints of the dead adds profoundly to the humanitarian character of human identification as part of the management of the dead.

\section{Acknowledgement}

The authors would like to thank the Director General of Health, Ministry of Health Malaysia for his permission to publish this study. We greatly acknowledged the tremendous support from the Director of the Hospital Kuala Lumpur (HKL) for providing us a platform to conduct research. The authors also would like to express our gratitude towards the Humanitarian and Human Rights Resource Center (HHRRC) from the American Academy of Forensic Sciences (AAFS) and the National Institute of Justice through their Forensic Technology Center of Excellence Program, RTI International for their generous research assistance in thisstudy.Special thanks to the Kridentia Tech SdnBhd for their unconditional contribution in partaking in the study. Finally, we would like to advance appreciation to all personnel of Department of Forensic Medicine, HKL whom have helped with the experimental research work and data collection.

\section{References}

[1]. Khoo LS, Lai PS, Saidin MH, Noor Z, Mahmood MS. Cling film plastic wrap: An innovation for dead body packaging, preservation and transportation by first responders as a replacement for cadaver body bag in large scale disasters. Forensic Sci Int. 2018 Apr; 285:50-57. Pubmed PMID: 29433011.

[2]. Saini M, Kapoor AK. Biometrics in forensic identification: applications and challenges. J Forensic Med. 2016 May;1(108):2.

[3]. Y Faridah, N Haidawati, A K Kushsairy, SI Safie, S Khan, TS Gunawan, Fingerprint biometric systems. Trends Bioinformatics. (2016) 9: 52-58.

[4]. Lee P. Prints charming: how fingerprints are trailblazing mainstream biometrics. Biometric Technology Today. 2017 Apr 1;2017(4):8-11.

[5]. HKL/FOR/AK-01, Reception of Dead Body from Ward within Hospital (Amendment 2016).

[6]. Official web portal, National Registration Department, Ministry of Home Affairs, Malaysia, https://www.jpn.gov.my/en/. Access date: 17 November 2020

[7]. Cocks K, Torgerson DJ. Sample size calculations for pilot randomized trials: a confidence interval approach. J Clin Epidemiol. 2013 Feb;66(2):197-201. Pubmed PMID: 23195919.

[8]. Whitehead AL, Julious SA, Cooper CL, Campbell MJ. Estimating the sample size for a pilot randomised trial to minimise the overall trial sample size for the external pilot and main trial for a continuous outcome variable. Stat Methods Med Res. 2016 Jun;25(3):1057-73. Pubmed PMID: 26092476.

[9]. S Lemeshow, D Hosmer, J Klar, S Lwanga, Adequacy of sample size in health studies, World Health Organization (1980).

[10]. INTERPOL, Disaster Victim Identification DVI Guide (2018).

[11]. Johnson BT, Riemen JAJM. Digital capture of fingerprints in a disaster victim identification setting: a review and case study. Forensic Sci Res. 2018 Nov 13;4(4):293-302. Pubmed PMID: 32002488.

[12]. Kaushal N, Kaushal P. Human identification and fingerprints: a review. J Biomet Biostat. 2011 Nov;2(123):2.

[13]. Nayak VC, Rastogi P, Kanchan T, Lobo SW, Yoganarasimha K, Nayak S, Rao NG, et al. Sex differences from fingerprint ridge density in the Indian population. J Forensic Leg Med. 2010 Feb;17(2):84-6. Pubmed PMID: 20129427.

[14]. Kawagoe M, Tojo A. Fingerprint pattern classification. Pattern recognition. 1984 Jan 1;17(3):295-303.

[15]. Moayer B, Fu KS. A syntactic approach to fingerprint pattern recognition. Pattern recognition. 1975 Jun 1;7(1-2):1-23.

[16]. N Kaushal, Fingerprints: Historical Background and Future Trends. The Internet Journal of Forensic Science. 4 (2009) 1-5.

[17]. A. Tiwari,How does a fingerprint scanner work - The Application of Biometrics (2016).

[18]. Criminal Procedure Code 1935(Amended 2012), (Act 593), (2012).

[19]. Schou MP, Knudsen PJ. The Danish Disaster Victim Identification effort in the Thai tsunami: organisation and results. Forensic Sci Med Pathol. 2012 Jun;8(2):125-30. Pubmed PMID: 22113326.

[20]. Schuller-Götzburg P, Suchanek J. Forensic odontologists successfully identify tsunami victims in Phuket, Thailand. Forensic Sci Int. 2007 Sep 13;171(23):204-7. Pubmed PMID: 17029859. 
[21]. De Valck E. Major incident response: collecting ante-mortem data. Forensic Sci Int. 2006 May 15;159 Suppl 1:S15-9. Pubmed PMID: 16546337.

[22]. Gahr B, Drewitz M, Vöth R, Ritz-Timme S. Quality improvement of fingerprints of decayed corpses by local thanatopractical processing (Thanatoprint). GMS Interdiscip Plast Reconstr Surg DGPW. 2013 Nov 5;2:Doc15. Pubmed PMID: 26504706.

[23]. Khoo LS, Hasmi AH, Mahmood MS, Vanezis P. Underwater DVI: Simple fingerprint technique for positive identification. Forensic Sci Int. 2016 Sep;266:e4-e9. Pubmed PMID: 27567043.
[24]. ICRC, Management of Dead Bodies after Disasters: A Field Manual for First Responders, Second edition 2016; 23-25.

[25]. Khoo LS, Mahmood MS. Application of facial recognition technology on identification of the dead during large scale disasters. Forensic Sci Int Synerg. 2020 Aug 7;2:238-239. Pubmed PMID: 32885161.

[26]. Cordner S, Tidball-Binz M. Humanitarian forensic action - Its origins and future. Forensic Sci Int. 2017 Oct;279:65-71. Pubmed PMID: 28846915. 